\title{
Thought and language disorders in very early onset schizophrenia, schizoaffective disorder and bipolar disorder
}

\author{
Telma Pantano1, Lee Fu I1, Eliana Curatolo¹, Camila Bertini Martins1,2, Helio Elkis ${ }^{1}$ \\ 1 Department and Institute of Psychiatry, University of São Paulo Medical School (FMUSP), São Paulo, SP, Brazil. \\ 2 Federal University of São Paulo (Unifesp), São José dos Campos, SP, Brazil.
}

Received: 8/6/2016 - Accepted: 5/9/2016

DOl: 10.1590/0101-60830000000087

\begin{abstract}
Background: Thought and language disorders are main features of adults with schizophrenia and bipolar disorders however studies on such abnormalities are scant in young patients with very early onset psychosis (VEOS). The aim of the present study is to assess the relationship between language and thought disorders in patients with very early onset schizophrenia (SCZ), schizoaffective disorders (SCA) and bipolar disorders (BD). Method: Forty-one patients (18 SCZ, 16 BD, and 7 SCA) with mean age less than 15 years old were assessed through a series of neurocognitive and psycholinguistic tests, including the Thought, Language and Communication Scale (TLC). Results: SCZ group performed worse in all tests as well as the TLC, followed by SCA and BD groups respectively. Thought disorders were related to deficits in executive functioning and semantic processing, and the metaphors' test was the best predictor of TLC functioning. Discussion: TD in SCZ, SCA and BD are one of the most important features in patients with VEOS and that the evaluation of metaphor comprehension can be an important instrument in the early detection of this disorder.
\end{abstract}

Pantano T et al. / Arch Clin Psychiatry. 2016;43(4):67-73

Keywords: Thought disorders, language, schizophrenia, bipolar disorder, schizoaffective disorder.

\section{Introduction}

Schizophrenia is a psychotic disorder which affects $1 \%$ of adult population and usually begins in late adolescence or early adulthood. When the disorder begins in early adolescence (under 18 years old) it is termed early onset schizophrenia (EOS), with some cases beginning even earlier (under 14 years old) and called child onset schizophrenia (COS) or very early onset schizophrenia (VEOS) ${ }^{1-3}$

EOS is a rare condition and it is estimated to account for less than $4 \%$ of the cases of schizophrenia 4 . Delusions, hallucinations and thought disorders are considered prominent psychopathological features of EOS 1,4 similar to adult onset schizophrenia.

Particularly formal thought disorders are also considered one main characteristic of psychotic manifestations in children or adolescents ${ }^{1}$. However such abnormalities are rarely studied in pediatric psychosis, especially in patients with early onset schizophrenia (VEOS) ${ }^{1}$.

All these thought impairments are also present in patients with bipolar disorders ${ }^{5-7}$ and to date there is only a few descriptions of such abnormalities in patients with early or very early onset of these disorders.

In fact thought disorders can be regarded as a failure to maintain a speech plan and thus encompassing a great number of abnormalities of logical sequencing of ideas however the connection between language and these disorders is not clearly established. Our hypothesis is that thought disorders are present in patients with VEOS and BD in different degrees of severity and are related to neuropsychological and language impairments.

The aim of the present study is to compare the performance of patients with schizophrenia (SCZ) or schizoaffective disorders (SCA) with patients with bipolar disorders (BD) in terms of thought disorders. Secondary objectives include investigating the relationship between thought disorders and neuropsychological performance, especially language disorders, using a battery of tests adapted to Brazilian Portuguese language.

\section{Methods}

\section{Sample}

Forty-one patients with psychotic symptoms (18 with diagnosis of the schizophrenia, 16 with bipolar disorders and 7 with schizoaffective disorder) were recruited for treatment at outpatient clinic of the Child and Adolescent Psychiatric Clinic of the Institute of Psychiatry of the University of São Paulo General Hospital (IPq) in São Paulo, Brazil. The study had the approval of the local Institutional Review Board of University of São Paulo General Hospital CAPPESQ $n^{\circ} 0088 / 07$ and families signed an informed consent for the participation in the study. To take part in the study patients had to be clinically stable after the medical treatment but which not received the language or cognition rehabilitation.

All the patients had between 10 and 17 years old at baseline and at the time of language and cognition evaluation. Patients were excluded if they had a previous or current history substance abuse, organic brain disease or marked intellectual impairment. For the control of the intellectual performance prior the illness all the patients had to be able to write and read and perform the four basic mathematical operations according to the school level and should not have any previous history of school performance impairment before the onset of the disorder. Additional information was obtained from chart reviews, parents' reports, school information and clinical interview.

\section{Clinical assessment}

Patients were interviewed by experienced child psychiatrists and met DSM-IV criteria for schizophrenia, schizoaffective disorder or bipolar disorder based on an interview using the Schedule for Affective Disorders and Schizophrenia for school age Children present and Lifetime version K-SADS 8 adapted into Portuguese. The K-Sads was applied by well-trained researchers with expertise in this instrument. 


\section{Neuropsychological and linguistic assessment}

All patients were evaluated through a battery comprising semantic and syntactic psycholinguistic tests, measures of executive functions and tests of general neurocognition. Such tests were usually performed within a week to the clinical interview for the DSM-IV and K-SADS diagnosis and administered by the same professional blind to the diagnosis. The battery was standardized to ensure the appropriate use of time, avoid tiredness of the patient and the application took approximately two sessions of 45 minutes.

\section{Semantic tests}

\section{MT-86b modified 9}

The protocol consists of different linguistics verbal, reading and writing tests to evaluate linguistic levels with or without visual images:

- Written comprehension: comprehension by the visual way word and phrases and the subsequent visual identification of this objects and situations;

- Reading: the patient must decode the words and talk them out loud.

\section{Peabody Picture Vocabulary test 10}

Oral comprehension: The subject is required to indicate which one of four pictures best describes a word spoken by an examiner. It is a thus of the comprehension of single words that refer to things or actions.

\section{Boston Naming Test ${ }^{11}$}

Is a test of visual confrontation naming in which the subject must retrieve (name).

\section{Semantic fluency 12}

The patient must produce as many examples as possible of animals (90 seconds). A score of "semantic efficiency" is related with the organization of semantic storage in the brain and the working memory tasks.

\section{Lexical decision ${ }^{13}$}

The patient distinguishes orally real words from nonexistent words with the oral and writes presentation.

\section{Metaphor's comprehension ${ }^{14}$}

Tests of oral comprehension of phrases with high symbolic significance.

\section{Syntactic tests}

\section{Syntactic Awareness 15}

The protocol consist of the differences abilities for evaluated syntactic awareness.

- Grammatical judgment: the patients should tell if there was something wrong in the phrases presented.

- Grammatical correction: the patients should to correct the phrases with wrong syntactic.

- Grammatical correction than phrases with grammatical and semantics inaccuracies: in this test the patients should to correct just the grammatical phrases but originally the phrases were with semantics inaccuracies which shouldn't be correct.

- Word's categorization: The patients should categorize the words in: substantive, adjective or verb.

\section{Executive function tests}

\section{Stroop test 16}

In the word task, the response is shown a card with the words BLUE, ROSE, GREEN and BROWN (printed in contrasting colors) arranged in four columns and six lines, to be read as quickly as possible ignoring their color. In the color task, a similar set of words is shown to the respondent who must how identify the color in which each word is printed. The Stroop effect is the difference (in seconds) between the time to complete the two tasks.

\section{Phonological fluency ${ }^{17}$}

In this test the respondent should produce as many words as possible beginning with the letters $\mathrm{T}$ (90 seconds). This test is thought to depend on the integrity of the left frontal lobe. The score is the total number of words generated excluding repetitions.

\section{More general cognitive function}

\section{Forward and reverse digit span ${ }^{18}$}

Forward digit span assess short-term memory. Respondents must repeat progressively longer sequences of digits read aloud to them at a rate of one digit per second. Span is defined as the longest string of digits correctly recalled at least once. Reverse span is administered in similar fashion but respondents must now reproduce the sequence in the reverse of the presentation order. This task involves manipulation of information as well as simple reiteration of it, and is often cited as a measure of working memory ${ }^{19}$.

\section{Speed processing}

The time for realization of each linguistic test (semantic and syntactic) was measured and added.

\section{Thought, Language and Communication Scale (TLC20)}

The Thought, Language, and Communication Scale (TLC) was used for language and thought evaluation. All the patients completed a detailed clinical interview lasting approximately 1 hour.

\section{Data analysis}

The descriptive statistics for continuous variables were mean and standard deviation; and for categorical variables, proportions. The relationship between the groups and categorical variables were analyzed by Chi Square Tests. For the continuous variables, the groups were compared using a series of ANOVA and Post Hoc Tests (Tukey), with normality tested using the KolmogorovSmirnov and Levene tests. The relationship between TLC and the others tests was explored by linear regression. The relationship among the tests was analyzed by Pearson coefficient. The significance was set to 0.05 .

\section{Results}

\section{Demographic and Clinical characteristics between the groups SCZ, BD, SCA}

There were no significant differences between groups in respect of age, gender, years of education and number of previous hospitalization. However in terms of duration of illness there was a significant difference between groups $(F=3.77, p=0.03)$, with bipolar patients showing an earlier age of onset (Table 1). 
Table 1. Demographic and clinical characteristics of the three diagnostic groups

\begin{tabular}{|c|c|c|c|c|}
\hline & $\operatorname{SCZ}(N=19)$ & $B D(N=16)$ & SCA $(N=7)$ & Test, $p$ \\
\hline Age - Mean (SD) & $15,9(1,63)$ & $14,7(2,47)$ & $15,8(1,87)$ & $F=1.67,0.20$ \\
\hline Gender & $11(\mathrm{M})$ & $10(\mathrm{M})$ & $5(\mathrm{M})$ & \multirow[t]{2}{*}{$X^{2}=0.401,0.818$} \\
\hline Male (M), female (F) & $8(\mathrm{~F})$ & $6(\mathrm{~F})$ & $2(\mathrm{~F})$ & \\
\hline Years of education & $7,47(2,04)$ & $7(1,83)$ & $7,29(1,7)$ & $\mathrm{F}=0.27,0.77$ \\
\hline Age of onset & $11,37(3,34)$ & $8,37(4,65)$ & $12,43(3,04)$ & $F=3.77,0.032$ \\
\hline Duration of illness & $4,58(2,89)$ & $6,37(3,94)$ & $3,40(1,99)$ & $\mathrm{F}=2.44,0.10$ \\
\hline \multicolumn{5}{|l|}{ Antipsychotic 1 (Main) } \\
\hline First-generation & 0 & 0 & $1(14.3 \%)$ & \multirow[t]{2}{*}{$X^{2}=21.18, p=0.048$} \\
\hline Second-generation & $19(100 \%)$ & $16(100 \%)$ & $6(85.7 \%)$ & \\
\hline \multicolumn{5}{|l|}{ Antipsychotic 2 (add-on) } \\
\hline First-generation & $1(5.3 \%)$ & 0 & 0 & \multirow[t]{2}{*}{$X^{2}=17.48, p=0.064$} \\
\hline Second-generation & $1(5.3 \%)$ & $3(18.8 \%)$ & $3(42.8 \%)$ & \\
\hline Antipsychotic monotherapy & $17(89.5 \%)$ & $13(81.3 \%)$ & $3(42.8 \%)$ & \\
\hline Mean dose (in Chlorpromazine equivalents) & 647 mg (sd 530) & 322 mg (sd314) & $454 \mathrm{mg}$ (sd 199) & $F=2.52, p=0.094$ \\
\hline \multicolumn{5}{|l|}{ Mood stabilizer 1} \\
\hline Lithium & $1(5.3 \%)$ & $5(31.3 \%)$ & $1(14.3 \%)$ & \multirow[t]{2}{*}{$X^{2}=10.13, p=0.04$} \\
\hline Anticonvulsant & $4(21 \%)$ & $6(37.5 \%)$ & 0 & \\
\hline \multicolumn{5}{|l|}{$\begin{array}{l}\text { Mood stabilizer } 2 \\
\text { (add-on) }\end{array}$} \\
\hline Lithium & 0 & 0 & 0 & \multirow[t]{2}{*}{$X^{2}=2.43, p=0.3$} \\
\hline Anticonvulsant & 0 & $1(6.3 \%)$ & $1(14.3 \%)$ & \\
\hline Mood stabilizer monotherapy & $5(26.3 \%)$ & $10(62.5 \%)$ & 0 & \\
\hline \multicolumn{5}{|l|}{ Antidepressant monotherapy } \\
\hline SSPA & $3(15.8 \%)$ & $2(12.5 \%)$ & $3(42.8 \%)$ & \multirow{2}{*}{$X^{2}=5.96, p=0.20$} \\
\hline Dual agent & $1(5.3 \%)$ & 0 & $1(14.3 \%)$ & \\
\hline
\end{tabular}

SCZ: schizophrenia; BD: bipolar disorder; SCA: schizoaffective disorder.

The medications in use by the subjects were classified according to the following psychopharmacological characteristics: antipsychotics (first and second generation) with their respective total mean dose expressed in chlorpromazine equivalents, types of mood stabilizer (lithium or anticonvulsants) and antidepressant prescribed (selective serotonin receptor agonist or dual agent). All the patients of the sample were receiving antipsychotics regardless of the diagnosis. The majority of the subjects were treated with antipsychotic monotherapy, with a second generation antipsychotic as the main or secondary treatment. The mean dose of antipsychotics (expressed in Chlorpromazine equivalents) was not significantly different between the groups $(\mathrm{F}=2.52, \mathrm{p}=0.094)$, but mood stabilizers were predominantly used by patients with $\mathrm{BD}$ than patients with SCZ or SCA $\left(\mathrm{X}^{2}=12.74, \mathrm{p}=0.047\right)$. The three groups showed no difference in terms of use of antidepressants $\left(\mathrm{X}^{2}=5.96\right.$, $0=0.20)$ (Table 1$)$.

We found no significant differences between groups in respect of the frequency of symptoms such as affective blunting, delusions, olfactory hallucinations, tactile hallucinations, disorganized speech and disorganized behavior. Patients with SCZ showed significantly higher frequencies of visual and auditory hallucinations when compared with SCA or BD (Table 2).

\section{Language and thoughts outcomes}

Table 3 displays the multiple comparisons of semantic and syntactic tests between diagnostic groups showed that, when compared with patients with BD, patients with SCZ showed a significantly poorer performance in terms of written comprehension $(p=0.006)$, oral comprehension $(\mathrm{p}=0.041)$, naming $(\mathrm{p}=0.001)$, semantic fluency $(\mathrm{p}=0.006)$, lexical decision $(\mathrm{p}=0.003)$, metaphor $(\mathrm{p}=$ $0.000)$, grammatical judgment $(\mathrm{p}=0.030)$, grammatical correction with semantics inaccuracies (0.005) and word's categorization $(\mathrm{p}=0.000)$. In terms of reading abilities and grammatical corrections both groups showed no significant differences. Patients with diagnostic of SCA had no substantial differences in terms of the tests previously described when compared with patients with BD or SCZ.

\section{Neurocognitive, executive function and psycholinguistic tests}

As can be observed by Table 4 the performance between the groups SCZ, BD and SCA showed no statistical significant differences in terms of the Stroop factor, phonological fluency, digit spam - forward and speed processing. However significant statistical differences between groups were found in terms of the of the TLC scale $(\mathrm{F}=18.73, \mathrm{p}=0.000)$ and digit spam - reverse $(\mathrm{F}=5.46, \mathrm{p}=$ 0.008 ). The multiple comparisons between the three groups showed that the SCZ had a poorer performance as compared with patients with BD in terms of the TLC $(\mathrm{p}=0.000)$, phonological fluency $(\mathrm{p}=0.056)$ and digit-spam reverse $(\mathrm{p}=0.019)$. No significant differences between the performance of the comparison between $\mathrm{BD}$ and SCA group as BD and SCA group.

\section{Predictors of TLC}

The relationship between total thought disorder, as measured by the TLC scale, and semantic, syntactic, neurocognitive, executive and psycholinguistic functions and was further examined using multiple regression. Separate stepwise analyses were performed with total TLC as the dependent variable and each of the above tests as predictor variables. As can be seen in Table 5 no relationship was found between TLC and any of linguistic or neurocognitive test except for the metaphor which showed to be the strongest predictor of thought disorder impairment in this population. 
Table 2. Frequency of K-SADS Psychopathology by diagnostic groups

\begin{tabular}{|l|c|c|c|c|}
\hline & SCZ & BD & SCA & Chi Square, $p$ \\
\hline Affective blunting & 1 & 0 & 1 & $2.21,0.33$ \\
\hline Delusions & 9 & 3 & 3 & $3.28,0.19$ \\
\hline Auditory hallucinations & 17 & 7 & 5 & $9.74,0.008$ \\
\hline Visual hallucinations & 14 & 4 & 0 & $9.25,0.01$ \\
\hline Olfactory hallucinations & 1 & 0 & 0 & $1.24,0.54$ \\
\hline Tactile hallucinations & 1 & 0 & 3 & $1.24,0.54$ \\
\hline Disorganized speech & 6 & 3 & 0 & $1.54,0.46$ \\
\hline Disorganized behavior & 1 & 1 & & $0.44,0.80$ \\
\hline
\end{tabular}

SCZ: schizophrenia; BD: bipolar disorder; SCA: schizoaffective disorder.

Table 3. Comparison of semantic and syntactic tests between diagnostic groups (post-hoc analysis)

\begin{tabular}{|c|c|c|c|c|c|c|}
\hline & & Mean (SD) & $F, p$ & Group Diagnostic & Mean Difference & Sig. \\
\hline \multirow{3}{*}{$\begin{array}{l}\text { Writing } \\
\text { Comprehension }\end{array}$} & SCZ & $9,26(3,16)$ & & SCZX BD & $-2,61$ & 0,006 \\
\hline & $\mathrm{BD}$ & $11,88(1,31)$ & $5.98, p=0.005$ & $\mathrm{BD} \times \mathrm{SCA}$ & 0,45 & 0,906 \\
\hline & SCA & $11,43(0,98)$ & & SCA X SCZ & 2,17 & 0,102 \\
\hline \multirow[t]{3}{*}{ Reading } & SCZ & $13,53(4,79)$ & & SCZXBD & $-2,54$ & 0,101 \\
\hline & $\mathrm{BD}$ & $16,06(2,11)$ & $2.71, p=0.079$ & BD X SCA & $-0,08$ & 0,999 \\
\hline & SCA & $16,14(1,35)$ & & SCA X SCZ & 2,62 & 0,230 \\
\hline \multirow[t]{3}{*}{ Oral Comprehension } & SCZ & $17,05(4,06)$ & & SCZXBD & $-2,64$ & 0,041 \\
\hline & $\mathrm{BD}$ & $19,69(2,06)$ & $3.21, p=0.051$ & $\mathrm{BD} \times \mathrm{SCA}$ & 1,12 & 0,706 \\
\hline & SCA & $18,57(1,27)$ & & SCA X SCZ & 1,52 & 0,511 \\
\hline \multirow[t]{3}{*}{ Naming } & SCZ & $11,37(2,19)$ & & SCZXBD & $-2,51$ & 0,001 \\
\hline & $\mathrm{BD}$ & $13,88(1,25)$ & $7.34, p=0.002$ & BD X SCA & 1,73 & 0,134 \\
\hline & SCA & $12,14(2,48)$ & & SCAXSCZ & 0,77 & 0,642 \\
\hline \multirow[t]{3}{*}{ Semantic Fluency } & SCZ & $11,63(4,65)$ & & SCZXBD & $-5,81^{*}$ & 0,006 \\
\hline & $\mathrm{BD}$ & $17,44(5,55)$ & $5.87, p=0.006$ & BD X SCA & 1,01 & 0,905 \\
\hline & SCA & $16,43(5,91)$ & & SCA X SCZ & 4,80 & 0,107 \\
\hline \multirow[t]{3}{*}{ Lexical Decision } & SCZ & $13,05(2,46)$ & & SCZXBD & $-2,20$ & 0,003 \\
\hline & $\mathrm{BD}$ & $15,25(1,07)$ & $8.319, p=0.001$ & BD X SCA & $-0,32$ & 0,920 \\
\hline & SCA & $15,57(0,79)$ & & SCA X SCZ & $2,52^{*}$ & 0,009 \\
\hline \multirow[t]{3}{*}{ Metaphor } & SCZ & $3,21(3,63)$ & & SCZXBD & $-6,04^{*}$ & 0,000 \\
\hline & $\mathrm{BD}$ & $9,25(4,30)$ & $9.60, p=0.000$ & BD X SCA & 2,25 & 0,454 \\
\hline & SCA & $7,00(4,87)$ & & SCA X SCZ & 3,80 & 0,105 \\
\hline \multirow{3}{*}{$\begin{array}{l}\text { Grammatical } \\
\text { Judgment }\end{array}$} & SCZ & $16,83(3,68)$ & & SCZXBD & $-2,42$ & 0,030 \\
\hline & $\mathrm{BD}$ & $19,25(1,18)$ & $3.64, p=0.036$ & BD X SCA & 0,75 & 0,825 \\
\hline & SCA & $18,50(1,23)$ & & SCA X SCZ & 1,67 & 0,385 \\
\hline \multirow{3}{*}{$\begin{array}{l}\text { Grammatical } \\
\text { Correction }\end{array}$} & SCZ & $6,94(3,12)$ & & SCZXBD & $-1,49$ & 0,279 \\
\hline & $\mathrm{BD}$ & $8,44(2,48)$ & $1.22, p=0.030$ & BD X SCA & 0,60 & 0,894 \\
\hline & SCA & $7,83(2,56)$ & & SCAXSCZ & 0,89 & 0,780 \\
\hline \multirow{3}{*}{$\begin{array}{l}\text { Grammatical } \\
\text { correction with } \\
\text { semantics } \\
\text { inaccuracies }\end{array}$} & SCZ & $6,39(2,85)$ & & SCZXBD & $-2,49 *$ & 0,005 \\
\hline & $\mathrm{BD}$ & $8,88(1,41)$ & $5.99, p=0.006$ & BD X SCA & 0,54 & 0,859 \\
\hline & SCA & $8,33(0,82)$ & & SCAXSCZ & 1,94 & 0,148 \\
\hline \multirow[t]{3}{*}{ Word's categorization } & SCZ & $6,61(4,41)$ & & SCZXBD & $-6,20$ & 0,000 \\
\hline & $\mathrm{BD}$ & $12,81(3,31)$ & $11.611, p=0.000$ & BD $\times$ SCA & 0,65 & 0,938 \\
\hline & SCA & $12,17(4,02)$ & & SCA X SCZ & 5,56 & 0,013 \\
\hline
\end{tabular}


Table 4. Comparison of selected neurocognitive, executive and psycholinguistic tests between diagnostic groups (post-hoc analysis)

\begin{tabular}{|c|c|c|c|c|c|c|}
\hline & & Mean (SD) & $F, p$ & Group Diagnostic & Mean Difference & Sig. \\
\hline \multirow[t]{3}{*}{ TLC } & SCZ & $32,47(12,37)$ & 18.73. $p=0.000$ & SCZX BD & $20,54^{*}$ & 0,000 \\
\hline & $\mathrm{BD}$ & $11,94(7,73)$ & & $\mathrm{BD} \times \mathrm{SCA}$ & $-14,06^{*}$ & 0,009 \\
\hline & SCA & $26,00(5,97)$ & & SCAX SCZ & $-6,47$ & 0,316 \\
\hline \multirow[t]{3}{*}{ Stroop Factor } & SCZ & $16,11(13,98)$ & $1.72, p=0.193$ & SCZXBD & 2,61 & 0,770 \\
\hline & $\mathrm{BD}$ & $13,50(8,54)$ & & BD X SCA & 6,50 & 0,409 \\
\hline & SCA & $7,00(5,80)$ & & SCAX SCZ & $-9,11$ & 0,166 \\
\hline \multirow[t]{3}{*}{ Phonological Fluency } & $\mathrm{SCZ}$ & $6,89(4,83)$ & $3.13, p=0.055$ & SCZX BD & $-3,86$ & 0,056 \\
\hline & $\mathrm{BD}$ & $10,75(4,73)$ & & $\mathrm{BD} \times \mathrm{SCA}$ & 0,61 & 0,957 \\
\hline & SCA & $10,14(4,67)$ & & SCAX SCZ & 3,25 & 0,283 \\
\hline \multirow[t]{3}{*}{ Digit Spam - forward } & $\mathrm{SCZ}$ & $5,00(1,03)$ & $1.78, p=0.183$ & SCZX BD & $-0,69$ & 0,157 \\
\hline & $\mathrm{BD}$ & $5,69(1,08)$ & & $\mathrm{BD} \times \mathrm{SCA}$ & 0,41 & 0,684 \\
\hline & SCA & $5,29(1,11)$ & & SCAX SCZ & 0,29 & 0,819 \\
\hline \multirow[t]{3}{*}{ Digit Spam - reverse } & SCZ & $2,39(1,38)$ & $5.46, p=0.008$ & SCZXBD & $-1,24^{*}$ & 0,019 \\
\hline & $\mathrm{BD}$ & $3,63(1,31)$ & & BD X SCA & $-0,23$ & 0,914 \\
\hline & SCA & $3,86(0,69)$ & & SCAX SCZ & $1,47^{*}$ & 0,034 \\
\hline \multirow[t]{3}{*}{ Time Processing } & SCZ & $404,84(173,37)$ & $2.45, p=0.100$ & SCZXBD & 123,28 & 0,085 \\
\hline & $\mathrm{BD}$ & $281,56(155,06)$ & & BD X SCA & $-86,44$ & 0,488 \\
\hline & SCA & $368,00(166,99)$ & & SCAX SCZ & $-36,84$ & 0,870 \\
\hline
\end{tabular}

SCZ: schizophrenia; BD: bipolar disorder; SCA: schizoaffective disorder.

Table 5. Regression Analysis: Thought Language and Communication Scale (TLC) vs. tests (predictors)

\begin{tabular}{|l|c|c|c|}
\hline Tests & Beta & $\mathrm{t}$ & $\mathrm{p}$ \\
\hline Writing Comprehension & -0.008 & -.051 & 0.960 \\
\hline Reading & 0.055 & 0.375 & 0.710 \\
\hline Oral Comprehension & -0.064 & -0.448 & 0.657 \\
\hline Naming & -0.181 & -1.185 & 0.244 \\
\hline Semantic Fluency & -0.252 & -1.715 & 0.095 \\
\hline Lexical Decision & 0.123 & 0.802 & 0.428 \\
\hline Grammatical Judgment & -0.208 & -1.449 & 0.156 \\
\hline Grammatical Correction & 0.107 & 0.634 & 0.530 \\
\hline Grammatical correction with semantics inaccuracies & -0.221 & -1.354 & 0.184 \\
\hline Word's categorization & 0.116 & 0.592 & 0.558 \\
\hline Stroop Factor & -0.182 & -1.449 & 0.156 \\
\hline Phonological Fluency & 0.045 & -0.275 & 0.785 \\
\hline Digit Spam - forward & -0.252 & -1.941 & 0.060 \\
\hline Digit Spam - reverse & 0.085 & 0.596 & 0.555 \\
\hline Time processing & 0.040 & 0.252 & 0.802 \\
\hline Metaphor & -0.626 & -4.951 & 0.000 \\
\hline
\end{tabular}

Model $=F=24.514, p=0.000$.

\section{Discussion}

To our knowledge this is the first study which compared patients with very early onset schizophrenia, bipolar disorder and schizoaffective disorder in terms of language disorders and its relationship with cognitive impairment. We found that patients with schizophrenia showed the highest degree of severity of thought disorders when compared with patients with other diagnostic categories.

Our data provide evidence that in terms of neurocognitive functions patients with schizophrenia have shown the worst performance when compared with patients with bipolar disorders or schizoaffective disorders even if we consider that the population studied the onset of symptoms of each disease was higher for patients with BD than patients with SCZ or SCA. These results are in agreement with several studies which related schizophrenia and bipolar disorders ${ }^{21}$ and show a distribution as observed what is called the "continuum of the psychosis" hypothesis 22 with patients with schizophrenia showing the worst performance, patients with bipolar disorder with the best performance and schizoaffective disorder patients in an intermediate position.

Although patients with schizophrenia performed significantly poorly in terms of the most of the linguistic and cognitive tests as thought disorders the differences were not significant in tests of reading abilities, grammatical correction, attention, phonological fluency and digit spam probably because these abilities were learned before the onset of the illness. When considered the alteration with thought disorders the regression analyses identified the metaphor comprehension as the best predictor for this alteration.

It was observed that the metaphor comprehension is possibly associated with frontal and temporal lobe functioning and in the 
left hemisphere involving activation of the prefrontal and basal frontal cortex, the middle and inferior temporal gyri, the temporal pole, the parietal cortex and the precuneus. In the language comprehension, activation is also observed in the right hemisphere: prefrontal cortex, middle temporal gyrus, precuneus and posterior cingulated gyrus ${ }^{23}$.

Our findings are thus broadly congruent with a small but growing body of evidence linking thought disorder to a combination of executive dysfunction and circumscribed semantic impairment ${ }^{24-26}$ related to, but possibly not restricted to, access and adds the right hemisphere functioning as injured in the thought disorders.

It is important to observe that the metaphor's test (figurative language) has largely been ignored in the cognitive science investigation field in favor of the investigation of literal language ${ }^{27}$. The present finding that metaphor comprehension is the best indicator of the thought disorder's performance must be replicated in larger studies. A limitation of the present study is the small number of subjects due to the low prevalence of the disorders in pediatric populations. Our findings show that language disorder are directly related with thought disorders ${ }^{19,24,25,28-34}$ with the relationship semantic and inter hemispheric frontal lobe.

This is important since cognitive features of these disorders, such as language and thought abnormalities, should become an important guide to new classifications about the cognitive dysfunction to understand the relation between functional capacities of these illnesses ${ }^{35}$. However, little is known about the behavior of these alterations in early-onset psychotic disorders and this study intends to bring new data on this subject.

The present paper also provides evidence that in terms of severity of thought and language disorders that the distribution of the impairments favors the idea of the so called continuum of psychosis $^{36-38}$.

Caution is needed when interpreting the findings due to methodological limitations. First, the possibility of a recruitment bias stemming from the fairly demanding nature of some of the tests in our neuropsychological battery, with some patients possibly being judged as "not competent to complete" them. Secondly, the premorbid neurocognitive status could not be estimated since tests like the National Adult Reading Test (Nelson, 199118), which estimates the premorbid IQ, are not available in Portuguese and the actual IQ was not investigated. However based on the clinical judgment patients with intelligence deficits were not included. Additionally neuropsychological functions were assessed using specific tests in Portuguese but not through a standard neuropsychological battery.

In conclusion, our data provide evidence that thought disorder in schizophrenia, schizoaffective disorder and bipolar disorder are one of the most important features in patients with VEOS and that the evaluation of metaphor comprehension can be an important instrument in the early detection of this disorder. In the present study though disorders showed to be associated with impairment in tests such as metaphor are related to frontal lobe function as well as semantic tests which are related to dysfunction of right and left hemispheres. Further investigations of relationship between executive and semantic impairments with thought disorder in schizophrenia accompanied by neuroimaging findings in patients with very early onset psychosis are warranted.

\section{Declaration of interest}

This research received no funding.

\section{References}

1. Vyas NS, Patel NH, Puri BK. Neurobiology and phenotypic expression in early onset schizophrenia. Early Interv Psychiatry. 2011;5(1):3-14.

2. Mattai AK, Hill JL, Lenroot RK. Treatment of early-onset schizophrenia. Curr Opin Psychiatry. 2010;23(4):304-10.
3. Clemmensen L, Vernal DL, Steinhausen HC. A systematic review of the long-term outcome of early onset schizophrenia. BMC Psychiatry. 2012;12:150.

4. Remschmidt H, Theisen F. Early-onset schizophrenia. Neuropsychobiology. 2012;66(1):63-9.

5. Osher Y, Bersudsky Y. Thought disorder in euthymic bipolar patients: a possible endophenotype of bipolar affective disorder? J Nerv Ment Dis. 2007;195(10):857-60.

6. Jabben N, Arts B, Van Os J, Krabbendam L. Neurocognitive functioning as intermediary phenotype and predictor of psychosocial functioning across the psychosis continuum: studies in schizophrenia and bipolar disorder. J Clin Psychiatry. 2010;71(6):764-74.

7. Krishnan RR, Kraus MS, Keefe RS. Hierarchical temporal processing deficit model of reality distortion and psychoses. Mol Psychiatry. 2011;16(2):129-44.

8. Brasil, HHA. Desenvolvimento da versão brasileira da K-SADS-PL (Schedule for Affective Disorders and Schizophrenia for School Aged Children Present and Lifetime Version) e estudo de suas propriedades psicométricas [tese]. Pós-Graduação em Psiquiatria e Psicologia Médica da Universidade Federal de São Paulo-USP, São Paulo; 2003.

9. Nespoulous J, Lecours AR, Mehler J, Parente MA. Protocolo MontrealToulouse-86B modificado para avaliação de afasia. Montreal: Centre de Recherche du CHCN; 1992.

10. Dunn LM, Dunn ES. Peabody Picture Vocabulary Test: Revised. Technical Supplement. Circle Pines: American Guidance Service; 1981.

11. Kaplan EGH, Weintraub S, editors. The Boston Naming Test. Philadeplphia: Lea Febisher; 1983.

12. Rohrer D, Salmon DP, Wixted JT, Paulsen JS. The disparate effects of Alzheimer's disease and Huntington's disease on semantic memory Neuropsychology. 1999;13(3):381-8.

13. Ober BA, Shenaut GK. Lexical decision and priming in Alzheimer's disease. Neuropsychologia. 1988;26(2);273-86.

14. Bottini G, Corcoran R, Sterzi R, Paulesu E, Schenone P, Scarpa P, et al. The role of the right hemisphere in the interpretation of figurative aspects of language: a positron emission tomography activation study. Brain. 1994;117(6):1241-53.

15. Capovilla AGS, Capovilla FC, Soares JVT. Consciência sintática no ensino fundamental: correlações com consciência fonológica, vocabulário, leitura e escrita. PsicoUSF, 2004;9(1):39-47.

16. Stroop JR. Studies of interference n serial verbal reactions. J Exp Psychol. $1935 ; 18: 643-62$.

17. Spreen O, Benton AL, editor. Neurosensory Center Comprehensive Examination for Aphasia. Victoria, British Columbia: University of Victoria; 1969.

18. Nelson HE. The revised National Adult Reading Test - Test manual. Windsor: NFER-Nelson; 1991.

19. Stirling J, Hellewel, J, Blakey A, Deakin W. Thought disorder in schizophrenia is associated with both executive dysfunction and circumscribed impairments in semantic function. Psychol Med. 2006;36(4):475-84.

20. Andreasen NC. Scale for the assessment of thought, language, and communication (TLC). Schizophr Bull. 1986;12(3):473-82.

21. Cuesta MJ, Peralta V. Does formal thought disorder differ among patients with schizophrenic, schizophreniform and manic schizoaffective disorders? Schizophr Res. 1993;10(2):151-8.

22. Lawrie SM, Hall J, McIntosh AM, Owens DG, Johnstone EC. The 'continuum of psychosis': scientifically unproven and clinically impractical. Br J Psychiatry. 2010;197(6):423-5.

23. Bottini G, Corcoran R, Sterzi R, Paulesu E, Schenone P, Scarpa P, et al The role of the right hemisphere in the interpretation of figurative aspects of language. A positron emission tomography activation study. Brain. 1994;117 ( Pt 6):1241-53.

24. Kerns JG, Berembaum H. Cognitive impairments associated with formal thought disorder in people with schizophrenia. J Abnorm Psychol. 2002;111(2):211-24.

25. Covington MA, He C, Brown C, Naçi L, McClain JT, Fjordbak BS, et al. Schizophrenia and the structure of language: the linguist's view. Schizophr Res. 2005;77(1):85-98.

26. Asarnow JR, Tompson MC, McGrath EP. Annotation: childhood-onset schizophrenia: clinical and treatment issues. J Child Psychol Psychiatry. 2004;45(2):180-94.

27. Remberk B, Namysłowska I, Rybakowski F. Clinical and cognitive correlates of formal thought disorder in early onset schizophrenia. Neuro Endocrinol Lett. 2012;33(3):347-55. 
28. Mortimer AM, Mc Kenna PJ. Levels of explanation - symptoms, neuropsychological deficit and morphological abnormalities in schizophrenia. Psychol Med. 1994;24(3):541-5.

29. Goldberg TE, Aloia, MS, Gourovitch ML, Missar D, Pickar D, Weinberger DR. Cognitive substrates of thought disorder, I: the semantic system. Am J Psychiatry. 1998;155(12):1671-6.

30. Oh TM, McCarthy RA, McKenna PJ. Is there a schizophasia? A study applying the single case approach to formal thought disorder in schizophrenia. Neurocase. 2002;8(3):233-44.

31. Woodruff PW, Wright IC, Shuriquie N, Russouw H, Rushe T, Howard $\mathrm{RJ}$, et al. Structural brain abnormalities in male schizophrenics reflect fronto-temporal dissociation. Psychol Med. 1997;27(6):1257-66.

32. Jones SR, Fernyhough C. Thought as action: inner speech, self-monitoring, and auditory verbal hallucinations. Conscious Cogn. 2007;16(2):391-9.
33. Stephane M, Barton, S, Boutros NN. Auditory verbal hallucinations and dysfunction of the neural substrates of speech. Schizophr Res. 2001;50(1-2):61-78.

34. Shergill SS, Brammer MJ, Williams SC, Murray RM, McGuire PK. Mapping auditory hallucinations in schizophrenia using functional magnetic resonance imaging. Arch Gen Psychiatry. 2000;57(11):1033-8.

35. Teixeira A, Alvarenga-Silva $\mathrm{H}$. Clinic and therapeutics of first schizophrenia episode. Psiquiatria Biológica. 2003;11(3):91-7.

36. Crow T. Temporal lobe asymmetries as the key to the etiology of schizophrenia. Schizophr Bull. 1990;16(3):433-43.

37. Crow T. The 'big bang' theory of the origin of psychosis and the faculty of language. Schizophr Res. 2008;102(1-3):31-52.

38. Craddock N, Owen MJ Molecular genetics and the relationship between epilepsy and psychosis. Br J Psychiatry. 2010;197(1):75-6. 